\title{
Prävalenz, Kosten der Versorgung und Formen des dialysepflichtigen chronischen Nierenversagens in Deutschland: Vergleich der Dialyseversorgung innerhalb und außerhalb stationärer Pflegeeinrichtungen
}

\author{
Prevalence, Costs of Medical Treatment and Modalities of Dialysis- \\ dependent Chronic Renal Failure in Germany: Comparison of \\ Dialysis Care of Nursing Home Residents and in Outpatient Units
}

\section{(ㅇ) (1) $(8)$}

\author{
Autoren \\ Dennis Häckl ${ }^{1}$, Nils Kossack ${ }^{1}$, Tonio Schoenfelder ${ }^{1,2}$
}

Institute

1 Wissenschaftliches Institut für Gesundheitsökonomie und Gesundheitssystemforschung, (WIG2), Leipzig, Deutschland

2 Technische Universität Dresden, Lehrstuhl für Gesundheitswissenschaften/Public Health, Dresden, Deutschland

\section{Schlüsselwörter}

Nierenversagen, Dialyse, Heimdialyse, Hämodialyse, Peritonealdialyse, Routinedaten

\section{Key words}

renal failure, dialysis, home dialysis, hemodialysis, peritoneal dialysis, claims data

online publiziert $\quad 15.01 .2021$

\section{Bibliografie}

Gesundheitswesen 2021; 83: 818-828

DOI 10.1055/a-1330-7152

ISSN 0941-3790

(c) 2021. The Author(s).

This is an open access article published by Thieme under the terms of the Creative Commons Attribution-NonDerivative-NonCommercial-License, permitting copying and reproduction so long as the original work is given appropriate credit. Contents may not be used for commercial purpose, or adapted, remixed, transformed or built upon. (https://creativecommons. org/licenses/by-nc-nd/4.0/)

Georg Thieme Verlag KG, Rüdigerstraße 14,

70469 Stuttgart, Germany

Korrespondenzadresse

Tonio Schoenfelder

WIG2, Versorgungsforschung

Markt 8

04109 Leipzig

Deutschland

tonio.schoenfelder@wig2.de

\section{ZUSAMMENFASSUNG}

Ziel der Studie Für die Wohnbevölkerung Deutschlands stehen keine aktuellen Daten zur Prävalenz des chronischen Nierenversagens und den Versorgungskosten der Dialyse zur Verfügung. Das Ziel dieser Studie war die Erhebung der Prävalenz des dialysepflichtigen chronischen Nierenversagens und der Versorgungskosten der dialysepflichtigen Patienten sowie die Analyse von Unterschieden zwischen dialysepflichtigen Patienten in und außerhalb von stationären Pflegeeinrichtungen.

Methodik Analysiert wurden Abrechnungsdaten von gesetzlichen Krankenkassen aus der WIG2-Forschungsdatenbank, die rund 4,5 Mio. anonymisierte Versicherte enthält. Eingeschlossen wurden Patienten aller Altersgruppen, die 2017 aufgrund eines chronischen Nierenversagens eine ambulante Dialyse (EBM-Ziffer 13602) erhielten. Es erfolgte eine Hochrechnung identifizierter Versicherter für die GKV und BRD mit amtlichen Statistiken. Patienten, die in und außerhalb von stationären Pflegeeinrichtungen wohnhaft waren, wurden hinsichtlich Komorbiditäten, Leistungsinanspruchnahme und Kosten verglichen.

Ergebnisse Für 2017 wurden 87255 Dialysepflichtige in der GKV (^1 054 Personen/1 Mio. Einwohner, pmp) und 100.202 für die Wohnbevölkerung Deutschlands ( $\triangleq 1210 \mathrm{pmp}$ ) ermittelt. In stationären Pflegeeinrichtungen lebten rund $8 \%$ der Dialysepflichtigen (7676 Patienten). Bis 2040 ist von einem Anstieg Dialysepflichtiger von 20-23\% auf ca. 120 000-123000 zu rechnen und für Dialysepflichtige in stationären Pflegeeinrichtungen von ca. 37-44\% auf ca. 10500-11 000. Bei fast allen Dialysepflichtigen kommt die Hämodialyse zum Einsatz, die Peritonealdialyse wird kaum genutzt. Die durchschnittlichen jährlichen Versorgungskosten für dialysepflichtige Patienten, die nicht in einer Pflegeeinrichtung leben, betragen 53996 und $57205 €$ für solche in stationärer Pflege. Hieraus ergeben sich jährliche Versorgungskosten für die GKV von rund 4,73 Mrd. €. 
Schlussfolgerung In dieser Studie wurde die Prävalenz des chronischen Nierenversagens für Deutschland auf einer aktuellen Datengrundlage berechnet. Die Ergebnisse bewegen sich im Rahmen von Daten anderer europäischer Länder. Es wird ein beträchtlicher Anstieg der Dialysepflichtigen bis 2040 prognostiziert, insbesondere für jene die im Heim leben. Hierdurch ist mit einer weiteren Steigerung der Versorgungskosten zu rechnen. Die häufigste Dialyseform ist die Hämodialyse, bei Patienten sowohl innerhalb als auch außerhalb stationärer Pflegeeinrichtungen, die Peritonealdialyse wird hingegen in nur geringem Maße eingesetzt.

\section{ABSTRACT}

Study objectives Current estimates on the prevalence of chronic renal failure and the costs of dialysis in Germany's population are not available. The aim of this study was to assess the prevalence of dialysis-dependent chronic renal failure and treatment costs of dialysis-dependent patients in Germany as well as differences between those residing in nursing homes and those treated in outpatient units.

Methods Health insurance claims from 4.5 million anonymized patients in the WIG2 research database were analyzed. Patients of all ages who had received outpatient dialysis treatment for chronic renal failure in 2017 (using uniform value scale code 13602) were included. These insurance claims were extrapolated to the German statutory health insurance population and, using official statistics, further to the entire popu- lation of Germany. Data on comorbidities, use of health resources, and costs were compared among patients residing in nursing homes and those treated in outpatient units.

Results In 2017, there were 87,255 dialysis-dependent statutory health insurance patients ( 11,054 person/ 1 million population, pmp), and 100,202 in Germany's whole population ( $\cong 1,210 \mathrm{pmp}$ ). About $8 \%$ of dialysis-dependent patients $(n=7,676)$ were living in nursing homes. Our analyses predict an increase in dialysis-dependent patients of about 20-23\% (up to 120,000-123,000), with an increase of dialysis-dependent nursing home residents of $37-44 \%$ (up to $10,500-11,000$ ) by 2040 . Almost all dialysis-dependent patients were treated with hemodialysis; peritoneal dialysis was rarely observed. The average annual treatment costs for dialysis-dependent patients residing in nursing homes amounted to 57,205 Euro and 53,996 Euro per patient respectively, with total annual statutory health insurance treatment costs amounting to about 4.73 billion Euro in 2017.

Conclusion This study presents current estimates for dialysisdependent chronic renal failure in Germany. Our findings on prevalence are comparable to data from other European countries and suggest a considerable increase in dialysis dependency by 2040 , particularly for nursing home residents, resulting in a further increase in dialysis care costs. Hemodialysis was the most commonly used dialysis modality in patients living both in and out of nursing care facilities, with peritoneal dialysis rarely being used.

\section{Einleitung}

Chronische Nierenerkrankungen betrafen 2017 weltweit fast 700 Mio. Menschen und die Anzahl der Neuerkrankungen umfasst rund 20 Mio. Personen jährlich [1]. Schätzungen gehen bis 2040 von einer Erhöhung der Todesfälle um das 2,6-fache aus [2].

Chronische Nierenerkrankungen sind ein eigenständiger Risikofaktor für kardiovaskuläre Erkrankungen, können zu körperlichen und kognitiven Einschränkungen führen und die Lebenserwartung signifikant senken [3]. In fortgeschrittenem Stadium kann eine chronische Nierenerkrankung in einer terminalen Niereninsuffizienz resultieren [3]. Betroffene Patienten benötigen lebenslang ein Nierenersatzverfahren oder eine Nierentransplantation. Beide Behandlungen verursachen sehr hohe Kosten, sodass terminale Niereninsuffizienz als die kostenträchtigste chronische Erkrankung gilt [3]. Die jährlichen durchschnittlichen Aufwände für Dialysebehandlungen in Deutschland aus der Perspektive der gesetzlichen Krankenversicherung (GKV) liegen je nach zugrunde gelegter Datenquelle und betrachteter Population bei rund 32 000-58 $000 €$ je Patient [4-6]. Diese Kostenkalkulationen beruhen jedoch auf veralteten Daten, sodass die derzeitigen Kosten für dialysepflichtige Patienten hiervon abweichen können.

Bundesweite aktuelle Daten zur Prävalenz chronisch dialysepflichtiger Patienten, hiermit assoziierten Kosten und zur Entwicklung der Fallzahlen sind nicht verfügbar, weil Deutschland über kein nationales Register für Nierenerkrankungen verfügt [7]. Zwar existieren regionale Erhebungen zur chronischen Niereninsuffizienz (z. B. ActiFE [8], ESTHER [9], KORA [10], SHIP [11]), allerdings unterscheiden sich diese Studien in ihrer methodischen Vorgehensweise und hinsichtlich der eingeschlossenen Populationen [12,13], sodass die vorhandenen regionalen Daten nicht ohne Weiteres auf Gesamtdeutschland übertragbar sind. Daten auf Grundlage der Qualitätssicherungs-Richtlinie Dialyse (QSD-RL) geben zwar Aufschluss über die Anzahl der ständig dialysepflichtigen Patienten in der GKV, weisen jedoch keine bundesweiten Prävalenzen oder Kostenwerte aus [14].

Das Ziel dieser Studie war daher eine bundesweite Erhebung der Prävalenz und der Versorgungskosten von Patienten mit dialysepflichtigem chronischem Nierenversagen sowie eine Prognose der Patientenzahlen für die kommenden 2 Jahrzehnte. Studien zeigen, dass die Prävalenz von chronischen Nierenerkrankungen mit zunehmendem Alter stark ansteigt [15] und insbesondere Menschen, die in Pflegeheimen leben, eine besonders hohe Prävalenz haben. So beträgt der Anteil von Personen im Heim mit einer mittels der CKD-EPI-Formel gemessenen glomerulären Filtrationsrate (GFR) $<60 \mathrm{ml} / \mathrm{min}$ pro $1,73 \mathrm{~m}^{2}$ zwischen 35-50\% [16-19], wohingegen in der Altersgruppe der 65-74-Jährigen, die nicht in einem Heim leben, dieser Anteil bei nur rund 20-26\% liegt [12]. Menschen in Pflegeheimen haben häufig mehrere chronische Erkrankungen und erhalten daher eine Vielzahl unterschiedlicher Medikamente zur gleichen Zeit [17, 20]. Rund 50\% aller Arzneimittel oder deren Metabolite werden über die Niere ausgeschieden und $30 \%$ aller unerwünschten Arzneimittelwirkungen haben eine renale Ursache 
oder eine renale Auswirkung [21]. Aus diesem Grund wurden in dieser Studie dialysepflichtige Heimbewohner zusätzlich als Subpopulation betrachtet und dahingehend analysiert, ob sich deren Komorbiditäten und Inanspruchnahme von Gesundheitsleistungen von dialysepflichtigen Patienten, die nicht in einem Pflegeheim wohnhaft sind, unterscheiden.

\section{Methodik}

Die Kostenschätzung wurde aus der Perspektive der GKV vorgenommen. Für die Berechnungen wurden Krankenkassenabrechnungsdaten (Routinedaten) verwendet. Diese Vorgehensweise wurde gewählt, weil das für die Kostenberechnungen notwendige Preis-Mengengerüst bereits in den Routinedaten anhand jener Kosten abgebildet ist, die der GKV als Kostenträgerin entstehen [22]. Somit beruhen die vorgenommenen Kostenberechnungen auf einer einheitlichen Vorgehensweise und Datenquelle. Diese einheitliche Vorgehensweise hat den Vorteil, dass Erinnerungsfehler oder Schätzfehler zu einzelnen Kostenbestandteilen, die z. B. bei Nutzung von Fragebögen auftreten können, vermieden werden. Darüber hinaus können Ressourcenverbräuche und Kosten der Studienpopulation durch Nutzung von Routinedaten sehr umfassend und einheitlich definiert nach unterschiedlichen Sektoren analysiert werden (z. B. Krankenhausaufenthalte, ambulante Behandlungen, Medikation, Heil- und Hilfsmittel, usw.). Die vorhandene Datenbasis mit einer Grundpopulation von rund 4,5 Mio. Versicherten ermöglicht zudem den Aufgriff einer im Vergleich zu Primärdatenstudien großen Studienpopulation, die über einen Zeitraum von mehreren Jahren untersucht werden kann.

\section{Datenbasis}

Es wurde eine Routinedatenanalyse auf Grundlage der WIG2-Forschungsdatenbank durchgeführt, welche longitudinale Daten von rund 4,5 Mio. Versichertenanonymen der GKV aus dem gesamten Bundesgebiet enthält. Die Daten sind hinsichtlich der Alters-, Geschlechts- und Morbiditätsverteilung in Deutschland repräsentativ für die GKV-Versichertenpopulation und ermöglichen eine longitudinale Betrachtung der Versicherten von 2010 bis 2019 [23].

\section{Studienpopulation}

Es wurden Patienten eingeschlossen, die aufgrund einer Dialysepflicht im Kalenderjahr 2017 eine ambulante Dialyse erhielten. Aufgegriffen wurden die Patienten über eine über die 17 Kassenärztlichen Vereinigungen abgerechnete Zusatzpauschale für die kontinuierliche Betreuung eines dialysepflichtigen Patienten (EBM-Ziffer 13602). Es wurden keine Ausschlusskriterien definiert.

\section{Variablen}

Erhoben wurden Alter, Geschlecht, Komorbiditäten auf Grundlage des Elixhauser-Index [24], verordnete Arzneimittel, Dialyseform (Hämodialyse (HD), intermittierende Peritonealdialyse (IPD), Peritonealdialyse (PD)) sowie die Kosten für stationäre Behandlungen, ambulante Behandlungen, Medikamente, Fahrtkosten, Heil- und Hilfsmittel sowie sonstige Leistungen (ambulante und stationäre Rehabilitation, häusliche Krankenpflege, Palliativ- und Hospizversorgung). Krankengeld wurde nicht betrachtet.

\section{Datenanalyse}

Die Prävalenz dialysepflichtiger Patienten wurde über die Anzahl von dialysepflichtigen Patienten mit durchgeführter ambulanter Dialyse ermittelt. Die Prävalenz dialysepflichtiger Patienten, die in einem Pflegeheim leben, wurde durch Selektion von Patienten aus der Population aller Dialysepflichtigen mit Wohnort Pflegeheim ermittelt.

Für beiden Gruppen erfolgte eine Hochrechnung auf die GKVPopulation je Alters- und Geschlechtsgruppe der KM 6-Statistik (Versicherte der gesetzlichen Krankenversicherung nach Status, Alter, Wohnort und Kassenart) [25]. Zusätzlich erfolgte für beide Gruppen eine Hochrechnung auf Gesamtdeutschland [26]. Bei der Darstellung der Hochrechnungen erfolgte zur Berücksichtigung von Unsicherheiten die Angabe des $95 \%$-Konfidenzintervalls (95\%-KI).

Für die Projektion der Anzahl von Patienten mit Dialysepflicht im Jahr 2040 wurden Daten der 13. koordinierten Bevölkerungsvorausberechnung des statistischen Bundesamtes herangezogen [26]. Für die Hochrechnung wurden 2 Szenarien betrachtet:

Szenario 1 geht von einer konstanten Geburtenrate und einem moderaten Anstieg der Lebenserwartung aus. Für die Zuwanderung wird eine konservative Schätzung zugrunde gelegt: Geburtenrate 1,4 Kinder/Frau, Anstieg Lebenserwartung bei Geburt bis 2060 für Jungen auf 84,8 Jahre und für Mädchen auf 88,8 Jahre, langfristiger Wanderungssaldo 200 000/Jahr [26].

Szenario 2 geht von einer höheren Geburtenrate sowie einem stärkeren Anstieg der Lebenserwartung aus. Für die Zuwanderung wird eine konservative Schätzung zugrunde gelegt: Geburtenrate 1,6 Kinder/Frau, Anstieg Lebenserwartung bei Geburt bis 2060 für Jungen auf 86,7 Jahre bzw. für Mädchen auf 90,4 Jahre, langfristiger Wanderungssaldo 200 000/Jahr [26].

Zusätzlich erfolgte eine separate Analyse der Dialysepflichtigkeit im Zeitverlauf vor und nach Einzug in das Pflegeheim. Hierzu wurden jene Patienten betrachtet, die während ihres stationären Heimaufenthaltes in den Jahren 2013 bis 2017 mindestens einmal als dialysepflichtige Patienten im ambulanten Bereich behandelt wurden (EBM-Ziffer 13602). Als Indexquartal wurde das Quartal des Einzugs in das Pflegeheim gewertet. Bei der Analyse wurde ein Vorbetrachtungszeitraum von 8 Quartalen vor dem Indexquartal sowie ein Nachbetrachtungszeitraum von 8 Quartalen nach dem Indexquartal bestimmt. In die Auswertung flossen sowohl Patienten ein, die über jeweils 8 Quartale vor bzw. nach dem Indexquartal beobachtbar waren, als auch Daten von zensierten Patienten, die nicht den gesamten Zeitraum beobachtbar waren (z. B. Verstorbene, Kassenwechsler).

In den Analysen wurden Mittelwerte (MW) und Standardabweichungen (SD) berechnet. Für Gruppenvergleiche wurden der t-Test für kontinuierliche Variablen und der Chi-Quadrat-Test für kategoriale Variablen genutzt. Unterschiede wurden als statistisch signifikant betrachtet bei einer Irrtumswahrscheinlichkeit von $\alpha<0,05$ bei zweiseitigen Tests. 


\section{Ergebnisse}

\section{Prävalenz dialysepflichtiger Patienten}

Für das Kalenderjahr 2017 wurden 3578 Versicherte identifiziert. Dies entspricht 87255 (95\%-KI: 84 803-89 706) dialysepflichtigen Versicherten in der GKV und einem Anteil an allen Versicherten der GKV von 0,12\% (95\%-KI: 0,12\%-0,13\%). Für die Wohnbevölkerung Deutschlands wurden $N=100202$ Patienten berechnet $(\bumpeq 0,12 \%)$. Die höchste Prävalenz besteht in den Altersgruppen zwischen 75 und 84 Jahren ( $\triangleright$ Abb. 1 ).

Für das Kalenderjahr 2017 wurden 273 dialysepflichtige Versicherte, die in stationären Pflegeeinrichtungen leben, identifiziert. Dies entspricht 6965 (95\%-KI: 6808-7122) Personen und einem
Anteil an allen dialysepflichtigen Versicherten von 7,98\% (95\%-KI: 7,80\%-8,16\%). Für Gesamtdeutschland wurden N = 7676 Patienten berechnet (^7,67\%). Die höchste Prävalenz besteht bei beiden Geschlechtern in der Altersgruppe der 80-84-jährigen ( $\triangleright$ Abb. 2).

\section{Prognose Dialysepflichtiger für das Jahr 2040}

Für dialysepflichtige Patienten prognostizieren beide Szenarien einen Anstieg der Fallzahlen um 20-23\% von 100202 Patienten in 2017 auf 119779 bzw. 123409 in 2040. Für dialysepflichtige Patienten im Pflegeheim wurde ein Anstieg zwischen 37-44\% berechnet, was 10534 bzw. 11070 Patienten im Jahr 2040 entspräche ( Abb. 3).

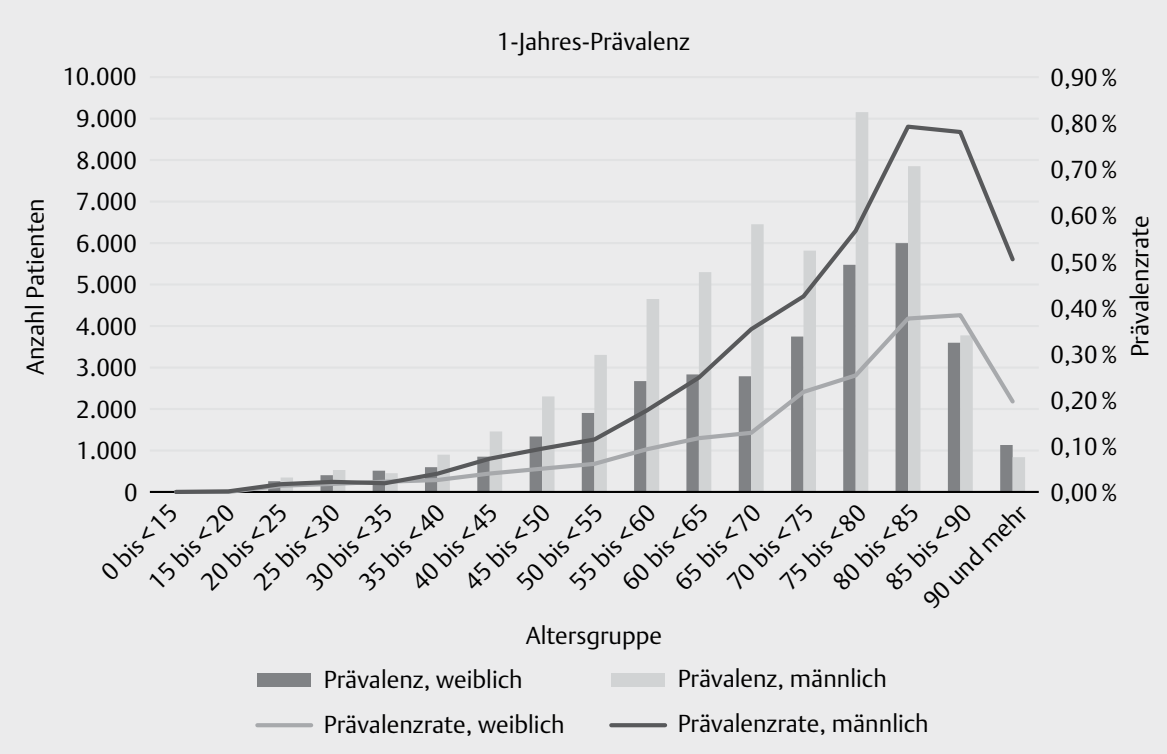

Abb. 1 Anzahl dialysepflichtiger Versicherter und altersgruppenspezifische Prävalenzraten in der GKV nach Alter und Geschlecht in 2017.

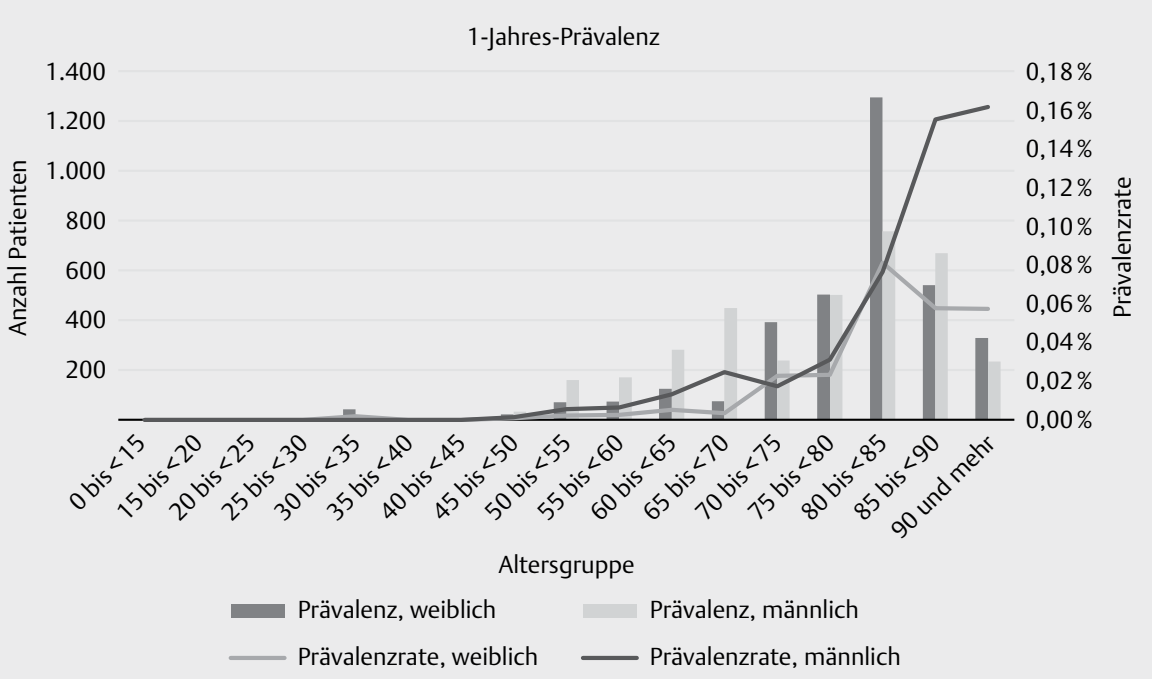

- Abb. 2 Anzahl dialysepflichtiger Versicherter im Pflegeheim und altersgruppenspezifische Prävalenzraten nach Alter und Geschlecht in 2017. 


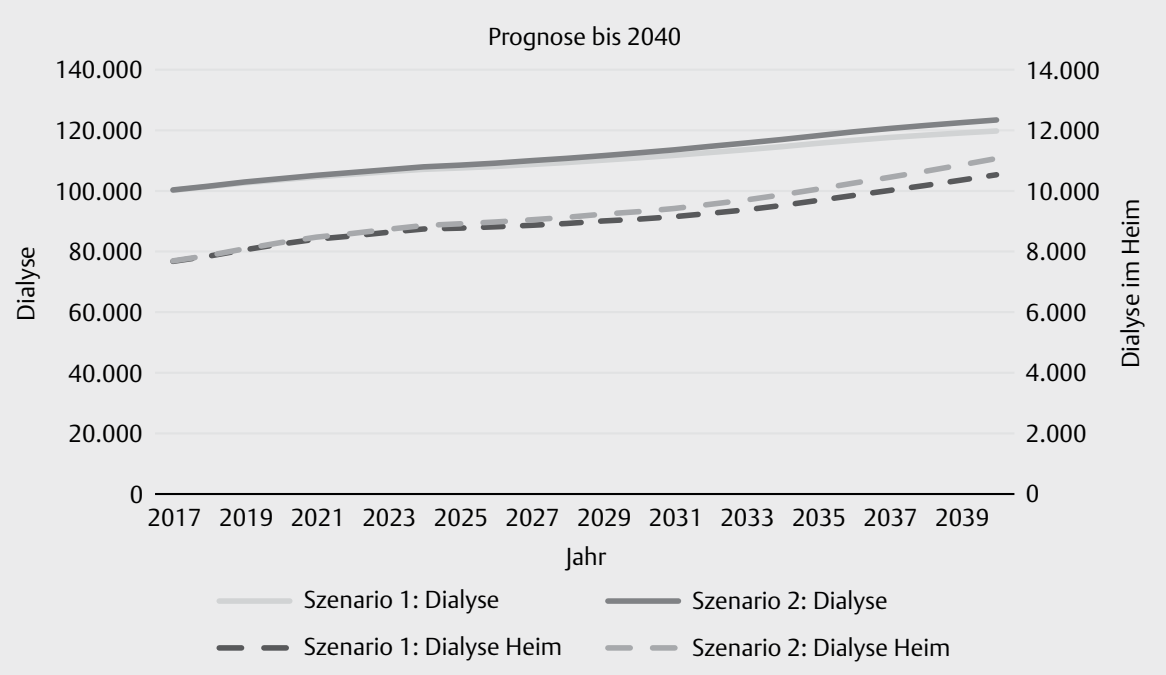

Abb. 3 Prognose der Anzahl dialysepflichtiger Patienten bis 2040.

\section{Unterschiede zwischen dialysepflichtigen Patienten}

Bei dialysepflichtigen Patienten außerhalb von stationären Pflegeeinrichtungen überwiegt der Anteil männlicher Patienten (61,8\%), in stationären Pflegeeinrichtungen sind beide Geschlechter gleich häufig vertreten. Dialysepflichtige Patienten in stationären Pflegeeinrichtungen sind durchschnittlich älter als dialysepflichtige Patienten außerhalb solcher Institutionen. Dialysepflichtige Frauen in stationären Pflegeheimen haben ein höheres Durchschnittsalter als dialysepflichtige Männer (ca. 2,6 Jahre) (๖ Tab. 1).

Die Mehrzahl der Patienten hatte typische Risikofaktoren für chronische Niereninsuffizienz wie Hypertonie, Herzinsuffizienz, Diabetes mellitus und Adipositas [3]. Dialysepflichtige Patienten in stationären Pflegeeinrichtungen weisen in den meisten Morbiditätsgruppen eine höhere Morbidität auf als Patienten, die nicht in stationären Pflegeeinrichtungen leben ( $\triangleright$ Tab. 1).

Bei den meisten der 10 am häufigsten verordneten Wirkstoffklassen für die Arzneimitteltherapie bestehen signifikante Unterschiede in der Verordnungshäufigkeit zwischen dialysepflichtigen Patienten im Pflegeheim und außerhalb ( $\triangleright$ Tab. 1). Am häufigsten verordnet wurden bei beiden Populationen Antianämika, Sulfonamide und Protonenpumpenhemmer. Die Unterschiede sind möglicherweise darauf zurückzuführen, dass die untersuchte Population im Heim wesentlich älter ist und eine höhere Morbiditätslast aufweist als Versicherte, die außerhalb eines Pflegeheims leben. So hatten Versicherte im Heim signifikant häufiger Diabetes mellitus, Herzinsuffizienz, Herzrhythmusstörungen oder periphere Gefäßkrankheiten ( $\triangleright$ Tab. 2).

\section{Dialyseform}

Am häufigsten in Anspruch genommen wurde die HD, die einen Anteil von $94,8 \%$ bei Versicherten außerhalb einer stationären Pflegeeinrichtung ausmachte bzw. 98,9\% bei Versicherten, die in einer stationären Pflegeeinrichtung lebten ( $\triangleright$ Tab. 1). Auf die PD entfielen insgesamt 9,3\% für Patienten außerhalb des Pflegeheimes und 4,4\% für Patienten in einem Pflegeheim. Die dargestell- ten Werte übersteigen die Summe von $100 \%$, weil im Verlauf eines Jahres die Dialyseform gewechselt werden kann und der Patientenaufgriff nicht zu einem Stichtag, sondern im Querschnitt eines Jahres erfolgte.

\section{Kosten}

Die durchschnittlichen jährlichen Versorgungskostenkosten für dialysepflichtige Patienten betragen $54223 €$ je Patient. Für die GKV ergeben sich hieraus Gesamtkosten von rund 4,73 Milliarden $€$ im Jahr 2017. Dialysepflichtige Patienten, die im Heim leben, weisen mit $57205 €$ rund $6 \%$ höhere Versorgungskosten auf, als Patienten, die nicht im Heim leben; deren durchschnittliche Versorgungskosten belaufen sich auf $53996 €$ ( $\triangleright$ Tab. 1). Dieser Kostenunterschied kommt im Wesentlichen durch höhere Krankenhauskosten ( 17070 vs. $13989 €$ ), Fahrtkosten (9778 vs. $6397 €$ ) und Kosten für Heil- und Hilfsmittel (2 267 vs. $1202 €$ ) auf Seiten der Heimpatienten zustande, wobei die Kosten für die Blutreinigung (Sachkosten für ambulant durchgeführte extrakorporale Blutreinigung, Kontenart 402) mit $17851 €$ im Vergleich zu rund $20926 €$ für Patienten außerhalb des Pflegeheims niedriger ausfallen.

Die durchschnittlichen jährlichen Versorgungskosten von Patienten mit Hämodialyse sind mit rund $54603 €$ signifikant höher als jene von Patienten mit Peritonealdialyse mit rund $46160 €$. Unterschiede bestanden insbesondere hinsichtlich Fahrtkosten und Krankenhauskosten, die bei Patienten mit Peritonealdialyse signifikant geringer waren, sowie der Versorgung durch Haus- und Fachärzte, Arzneimittel und Sachkosten für die Blutreinigung, die bei Patienten mit Peritonealdialyse signifikant höher waren ( $\triangleright$ Tab. 3).

\section{Betrachtung dialysepflichtiger Patienten im Heim im Längsschnitt}

Die Mehrzahl (69\%) der dialysepflichtigen Patienten, die im Heim leben, waren bereits vor ihrem Heimaufenthalt dialysepflichtig. Rund $18 \%$ der Patienten lebten bereits im Heim, als sie dialysepflichtig wurden, und $13 \%$ wurden im gleichen Quartal der Heim- 
aufnahme dialysepflichtig. Bei 98,65\% der Dialysepflichtigen wird zum Zeitpunkt des Einzugs in das Pflegeheim die HD eingesetzt, $1,10 \%$ entfallen auf die IPD und $0,25 \%$ auf die PD.

Bei Analyse des Vorbetrachtungszeitraums vor Heimeinzug ist ein Anstieg der Dialysepflicht bereits in den beiden Quartalen vor Beginn des Heimaufenthalts erkennbar. Rund $40 \%$ der im Pflegeheim lebenden Dialysepflichtigen wurden bereits zwei Jahre vor Beginn des Heimaufenthalts dialysiert ( $>$ Abb. 4). Nutzt man zum Aufgriff des Prädialysestadiums die Zusatzpauschale „kontinuierliche Betreuung eines chronisch niereninsuffizienten Patienten“ (EBM-Ziffer 13600), wird ersichtlich, dass nur knapp jeder 8. der später im Pflegeheim dialysepflichtigen Patienten vor Aufnahme in das Pflegeheim eine Prädialyse hatte.

\section{Diskussion}

Diese Studie untersuchte die Prävalenz und Versorgungskosten von Patienten mit chronischer Niereninsuffizienz und Dialysepflicht auf Basis von repräsentativen Abrechnungsdaten der GKV. Für 2017 wurde eine Prävalenz von 87255 dialysepflichtigen Patienten in der GKV und von 100202 dialysepflichtigen Patienten in Deutschland (inkl. privat Krankenversicherter) ermittelt. Dies entspricht rund 1054 Personen/1 Million Einwohner (person/million population, pmp) in der GKV bzw. 1210 pmp in der BRD.

Vergleichbare Daten zur Prävalenz des chronischen Nierenversagens für die gesamte BRD sind aktuell nicht verfügbar. Publizierte Daten für mehrere europäische Länder berichten für 2011 1031 pmp [27]. In der zitierten Studie wurde eine jährliche Steige-

- Tab. 1 Vergleich von Patienten, die in und außerhalb von stationären Pflegeeinrichtungen wohnhaft sind (2017).

\begin{tabular}{|c|c|c|c|}
\hline Kategorien & $\begin{array}{l}\text { Außerhalb von stationären } \\
\text { Pflegeeinrichtungen }(\mathrm{N}=3578)\end{array}$ & $\begin{array}{l}\text { In stationären } \\
\text { Pflegeeinrichtungen ( } N=273 \text { ) }\end{array}$ & p-Wert ${ }^{1}$ \\
\hline \multicolumn{4}{|l|}{ Geschlecht, \% (N) } \\
\hline Weibliche Patientinnen & $38,2 \%(1365)$ & $49,8 \%(136)$ & 0,0001 \\
\hline Männliche Patienten & $61,8 \%(2213)$ & $50,2 \%(137)$ & 0,0001 \\
\hline \multicolumn{4}{|l|}{ Alter (MW, SD) } \\
\hline Frauen & $68,1(15,7)$ & $79,1(11,2)$ & 0,0000 \\
\hline Männer & $67,6(14,6)$ & $76,5(11,5)$ & 0,0000 \\
\hline \multicolumn{4}{|l|}{ Komorbiditäten², \% (N) } \\
\hline Adipositas & $30,9 \%(1105)$ & $31,9 \%(87)$ & 0,7344 \\
\hline AIDS/HIV & $0,2 \%(7)$ & $0,0 \%(0)$ & 0,4645 \\
\hline Alkoholmissbrauch & $5,6 \%(199)$ & $9,7 \%(26)$ & 0,0071 \\
\hline Andere neurologische Erkrankungen & $9,8 \%(351)$ & $23,4 \%(64)$ & 0,0000 \\
\hline Blutungsanämie & $2,3 \%(84)$ & $3,3 \%(9)$ & 0,3248 \\
\hline Chronische pulmonare Erkrankungen & $31,6 \%(1129)$ & $39,4 \%(108)$ & 0,0063 \\
\hline Depression & $22,5 \%(804)$ & $31,8 \%(87)$ & 0,0004 \\
\hline Diabetes, kompliziert ${ }^{3}$ & $43,4 \%(1553)$ & $58,6 \%(160)$ & 0,0000 \\
\hline Diabetes, unkompliziert ${ }^{3}$ & $45,2 \%(1616)$ & $57,7 \%(157)$ & 0,0001 \\
\hline Drogenmissbrauch & $1,9 \%(69)$ & $2,2 \%(6)$ & 0,7562 \\
\hline Gewichtsverlust & $9,4 \%(335)$ & $19,0 \%(52)$ & 0,0000 \\
\hline Herzklappenfehler & $34,3 \%(1229)$ & $40,0 \%(109)$ & 0,0621 \\
\hline Herzrhythmusstörung & $43,1 \%(1541)$ & $59,3 \%(162)$ & 0,0000 \\
\hline Hypertonie, kompliziert ${ }^{3}$ & $68,4 \%(2447)$ & $67,3 \%(184)$ & 0,7344 \\
\hline Hypertonie, unkompliziert ${ }^{3}$ & $92,9 \%(3323)$ & $95,5 \%(261)$ & 0,0868 \\
\hline Hypothyreose & $23,1 \%(828)$ & $30,2 \%(83)$ & 0,0065 \\
\hline Koagulopathie & $15,0 \%(537)$ & $18,5 \%(51)$ & 0,1039 \\
\hline Kongestive Herzinsuffizienz & $51,5 \%(1843)$ & $69,7 \%(190)$ & 0,0000 \\
\hline Krankheiten des Lungenkreislaufes & $13,0 \%(466)$ & $14,3 \%(39)$ & 0,5516 \\
\hline Lähmung & $6,8 \%(244)$ & $16,8 \%(46)$ & 0,0000 \\
\hline Lebererkrankungen & $19,9 \%(711)$ & $20,8 \%(57)$ & 0,6879 \\
\hline Lymphom & $2,3 \%(83)$ & $0,3 \%(1)$ & 0,0332 \\
\hline Mangelanämie & $25,2 \%(903)$ & $24,6 \%(67)$ & 0,7986 \\
\hline Metastasierter Krebs & $4,3 \%(154)$ & $6,5 \%(18)$ & 0,0775 \\
\hline Nichtblutendes Magengeschwür & $3,6 \%(127)$ & $6,5 \%(18)$ & 0,0109 \\
\hline Periphere Gefäßkrankheiten & $51,7 \%(1848)$ & $66,8 \%(182)$ & 0,0000 \\
\hline Psychose & $0,9 \%(33)$ & $4,3 \%(12)$ & 0,0000 \\
\hline Rheumatoide Arthritis & $9,3 \%(331)$ & $8,4 \%(23)$ & 0,6488 \\
\hline Solider Tumor ohne Metastase & $18,0 \%(644)$ & $23,7 \%(65)$ & 0,0170 \\
\hline Störungen des Wasser- und Elektrolythaushaltes & $55,9 \%(2000)$ & $59,4 \%(162)$ & 0,2691 \\
\hline
\end{tabular}


Tab. 1 Fortsetzung.

\begin{tabular}{|c|c|c|c|}
\hline Kategorien & $\begin{array}{l}\text { Außerhalb von stationären } \\
\text { Pflegeeinrichtungen }(\mathrm{N}=3578)\end{array}$ & $\begin{array}{l}\text { In stationären } \\
\text { Pflegeeinrichtungen ( } N=273)\end{array}$ & p-Wert ${ }^{1}$ \\
\hline \multicolumn{4}{|l|}{ Arzneimitteltherapie $^{4}, \%(\mathrm{~N})$} \\
\hline \multicolumn{4}{|l|}{ Antianämika: } \\
\hline Eisen, parenterale Zubereitung & $68,0 \%(2434)$ & $66,3 \%(181)$ & 0,2030 \\
\hline Vitamin D and Analoga & $70,8 \%(2535)$ & $63,7 \%(174)$ & 0,0131 \\
\hline Andere Antianämika & $86,3 \%(3087)$ & $89,0 \%(243)$ & 0,5559 \\
\hline Sulfonamide & $80,4 \%(2875)$ & $80,6 \%(220)$ & 0,9253 \\
\hline Protonenpumpenhemmer & $69,2 \%(2475)$ & $79,1 \%(216)$ & 0,0006 \\
\hline $\begin{array}{l}\text { Mittel zur Behandlung der Hyperkaliämie und } \\
\text { Hyperphosphatämie }\end{array}$ & $68,3 \%(2444)$ & $48,4 \%(132)$ & 0,0000 \\
\hline Beta-Adrenozeptor-Antagonisten, selektiv & $65,7 \%(2350)$ & $52,4 \%(143)$ & 0,0000 \\
\hline Pyrazolone & $48,1 \%(1721)$ & $61,0 \%(169)$ & 0,0000 \\
\hline Dihydropyridin-Derivate & $47,2 \%(1690)$ & $34,8 \%(95)$ & 0,0001 \\
\hline HMG-CoA-Reduktasehemmer & $42,2 \%(1510)$ & $35,5 \%(97)$ & 0,0312 \\
\hline \multicolumn{4}{|l|}{ Dialyseform ${ }^{5}, \%(\mathrm{~N})$} \\
\hline Hämodialyse & $94,8 \%(3391)$ & $98,9 \%(270)$ & 0,0010 \\
\hline Peritonealdialyse & $7,5 \%(270)$ & $2,2 \%(6)$ & 0,6518 \\
\hline Intermittierende Peritonealdialyse & $1,8 \%(65)$ & $2,2 \%(6)$ & 0,0024 \\
\hline \multicolumn{4}{|c|}{$\begin{array}{l}\text { 1t-Test; }{ }^{2} \text { Komorbiditäten selektiert anhand des Elixhauser Index [18]; }{ }^{3} \text { Die Zuordnung einzelner Patienten zu den Komorbiditäten kann zustande } \\
\text { kommen, wenn der gleiche Arzt im Zeitverlauf unterschiedliche Diagnosen stellt oder unterschiedliche Ärzte unterschiedliche Diagnosen stellen. Die } \\
\text { Auftretenshäufigkeiten für die Diagnosevarianten (kompliziert, unkompliziert) des Diabetes mellitus und der Hypertonie summieren sich daher zu } \\
\text { mehr als } 100 \% \text { auf. }{ }^{4} \text { Dargestellt werden die } 10 \text { am häufigsten verordneten Wirkstoffklassen; }{ }^{5} \text { Werte übersteigen } 100 \% \text {, weil im Verlauf eines Jahres } \\
\text { die Dialyseform gewechselt werden kann und der Patientenaufgriff nicht zu einem Stichtag, sondern im Querschnitt eines Jahres erfolgte. MW = Mit- } \\
\text { telwert, SD = Standardabweichung. }\end{array}$} \\
\hline
\end{tabular}

- Tab. 2 Jährliche durchschnittliche Kosten dialysepflichtiger Patienten nach Sektoren (2017).

\begin{tabular}{|c|c|c|c|c|}
\hline Leistungen & $\begin{array}{l}\text { Gesamtpopulation, } \\
\text { (MW Kosten in } € \text { (SD), } \\
\text { [Median] }\end{array}$ & $\begin{array}{l}\text { Außerhalb von stationären } \\
\text { Pflegeeinrichtungen (MW Kosten } \\
\text { in } € \text { (SD), [Median]) }\end{array}$ & $\begin{array}{l}\text { In stationären Pflegeein- } \\
\text { richtungen (MW Kosten } \\
\text { in } €(S D) \text {, [Median]) }\end{array}$ & p-Wert ${ }^{1}$ \\
\hline Gesamtkosten & $\begin{array}{l}\text { 54223,73 (34461,41), } \\
{[48679,65]}\end{array}$ & $53996,23(34249,77),[48.114,24]$ & $\begin{array}{l}57205,37(37235,25), \\
55859,12]\end{array}$ & 0,1382 \\
\hline Haus- und Fachärzte & $\begin{array}{l}3439,31(1502,32), \\
{[3493,95]}\end{array}$ & $3458,07(1499,94),[3493,70]$ & $\begin{array}{l}3193,41(1533,44), \\
{[3502,01]}\end{array}$ & 0,0050 \\
\hline Zahnärzte & $163,06(418,60),[9,44]$ & 161,77 (399,46), $[18,48]$ & 180,00 (669,43), [0] & 0,4937 \\
\hline Krankenhaus & $\begin{array}{l}\text { 14206,98 (16966,57), } \\
{[6108,30]}\end{array}$ & $13988,54(26603,14),[5771,06]$ & $\begin{array}{l}17069,86(21533,39), \\
{[10871,51]}\end{array}$ & 0,0045 \\
\hline Arzneimittel & $\begin{array}{l}7449,52(26243,74), \\
{[5192,78]}\end{array}$ & 7567,23 (17 839,68), [5 220,25] & $\begin{array}{l}5906,79(5523,44), \\
{[4700,83]}\end{array}$ & 0,3143 \\
\hline $\begin{array}{l}\text { Sachkosten für Blutreinigung } \\
\text { (Kontenart 402) }\end{array}$ & $\begin{array}{l}20708,28(8539,33), \\
{[24751,96]}\end{array}$ & $20926,27(8467,12),[24792,27]$ & $\begin{array}{l}17851,37(9485,69), \\
{[21948,12]}\end{array}$ & 0,0000 \\
\hline Fahrtkosten & $\begin{array}{l}6636,36(6161,16), \\
{[5010,59]}\end{array}$ & 6396,63 (5 934,47), [4 806,09] & $\begin{array}{l}9778,30(9132,20), \\
{[8339,76]}\end{array}$ & 0,0000 \\
\hline Heil- und Hilfsmittel & $\begin{array}{l}1277,15(2752,00), \\
{[160,36]}\end{array}$ & 1201,61 (2711,93), [126,00] & $\begin{array}{l}2267,17(3277,11), \\
{[922,56]}\end{array}$ & 0,0000 \\
\hline $\begin{array}{l}\text { Sonstige Kosten (Rehabilitation, } \\
\text { häusliche Krankenpflege, } \\
\text { Palliativ- und Hospizversorgung) }\end{array}$ & 343,06 (892,80), [0] & 296,11 (812,65), [0] & 958,46 (1943,23), [0] & 0,0000 \\
\hline
\end{tabular}

rung der Prävalenz von 3,3\% in den zurückliegenden Jahren genannt. Bei Zugrundelegung dieser Steigerungsrate ergeben sich für das Jahr 20171254 pmp, was mit der in dieser Studie ermittelten Anzahl Dialysepflichtiger von 1210 pmp vergleichbar ist.
Hinsichtlich der Prävalenz des chronischen Nierenversagens in der GKV stimmen die ermittelten Zahlen annähernd mit Daten auf Grundlage der QSD-RL überein [14]. Dort wird die Zahl der ständig dialysepflichtigen Patienten mit HD oder PD für 2017 mit insge- 
• Tab. 3 Jährliche durchschnittliche Kosten der Hämodialyse im Vergleich zur Peritonealdialyse nach Sektoren (2017).

\begin{tabular}{|c|c|c|c|}
\hline Leistungen & $\begin{array}{l}\text { Hämodialyse }^{1} \\
\text { (MW Kosten in } € \text { (SD), [Median]) }\end{array}$ & $\begin{array}{l}\text { Peritonealdialyse }^{1} \\
\text { (MW Kosten in } € \text { (SD), [Median]) }\end{array}$ & p-Wert ${ }^{2}$ \\
\hline Gesamtkosten & $54603,66(33250,25),[49070,42]$ & $46160,70(56504,10),[38603,50]$ & 0,0033 \\
\hline Haus- und Fachärzte & $3416,25(1476,42),[3833,09]$ & $3928,79(2015,18),[3476,60]$ & 0,0001 \\
\hline Zahnärzte & $162,27(427,89 €),[0]$ & $179,80(321,46),[50,49]$ & 0,6199 \\
\hline Krankenhaus & $14482,31(26586,94),[6286,84]$ & $8363,79(16335,90),[3466,85]$ & 0,0052 \\
\hline Arzneimittel & $7341,80(14800,51),[5249,38]$ & $9735,50(47507,03),[3911,88]$ & 0,0960 \\
\hline $\begin{array}{l}\text { Sachkosten für Blutreinigung } \\
\text { (Kontenart 402) }\end{array}$ & 20622,43 (8335,29), [24691,56] & 22530,27 (8335,29), [26280,80] & 0,0060 \\
\hline Fahrtkosten & $6928,91(5201,11),[5274,56]$ & $427,76(1308,20),[56,00]$ & 0,0001 \\
\hline Heil- und Hilfsmittel & $1314,89(2769,55),[170,30]$ & 476,20 (1272,66), [0] & 0,0002 \\
\hline $\begin{array}{l}\text { Sonstige Kosten (Rehabilitation, } \\
\text { häusliche Krankenpflege, } \\
\text { Palliativ- und Hospizversorgung) }\end{array}$ & 334,79 (854,15), [0] & 518,59 (1251,54), [0] & 0,0115 \\
\hline \multicolumn{4}{|c|}{ 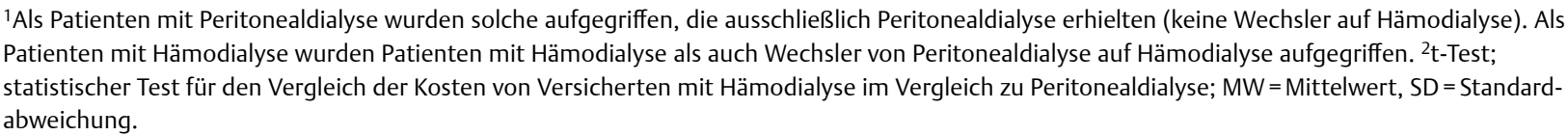 } \\
\hline
\end{tabular}

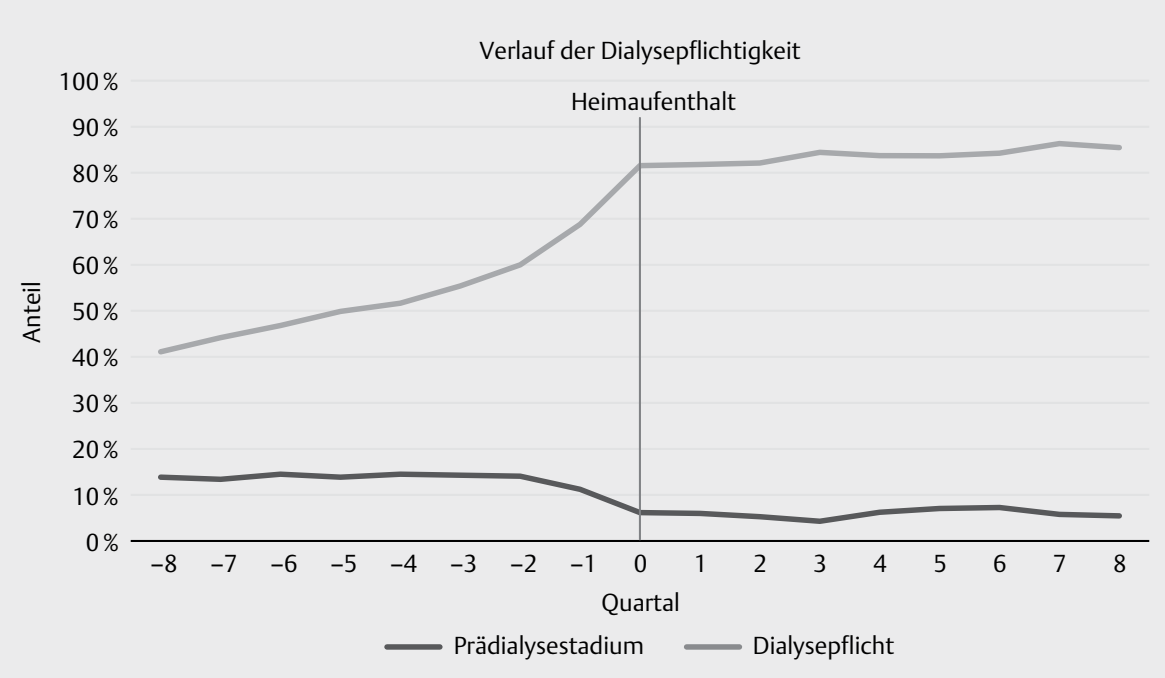

Abb. 4 Verlauf der Dialysepflichtigkeit 8 Quartale vor und nach der Heimaufnahme.

samt 86388 dialysepflichtigen Patienten in der GKV angegeben, was einer Abweichung zu denen in dieser Studie ermittelten Patientenzahlen von nur ca. $1 \%$ entspricht.

Weiterhin existieren zum Vergleich Daten aus regionalen Erhebungen zur Prävalenz von chronischen Nierenerkrankungen in Deutschland, die jedoch auch die Prävalenz für Stadien der chronischen Niereninsuffizienz ohne Dialysepflicht (GFR von $<60 \mathrm{ml} / \mathrm{min}$ pro $1,73 \mathrm{~m}^{2}$ ) ausweisen. So zeigen SHIP eine Prävalenz bei Patienten im Alter von 31-82 Jahren von 5,9\% (Norddeutschland) und KORA von 3,1\% (Augsburg) [12]. ActiFE (Ulm) und ESTHER berichte eine Prävalenz bei den 65-74-jährigen von 19,9-21,5\% [13]. Bei der Interpretation dieser Zahlen ist zu beachten, dass nicht alle Altersgruppen berücksichtigt werden. So schließt z. B. ESTHER Patienten nur bis 74 Jahren ein. Die Daten der vorliegenden Analyse zeigen jedoch, dass die Prävalenz von Patienten über 80 Jahren beträchtlich ist und insbesondere bei den weiblichen Dialysepflichtigen in der Altersgruppe der 80-84-jährigen am höchsten ist.

Für 2017 wurde eine Zahl von 7676 chronisch dialysepflichtige Patienten, die in stationären Pflegeheimen wohnhaft sind ( $\triangleq 93$ pmp), ermittelt. Studien aus Deutschland wurden nicht gefunden, sodass hierzu keine Daten als Vergleich herangezogen werden können.

Aus den Analysen geht hervor, dass der Anteil chronisch dialysepflichtiger Patienten mit zunehmendem Alter steigt. Daher ist zu erwarten, dass aufgrund der alternden Bevölkerung in den kommenden Jahren auch der Anteil der dialysepflichtigen Patienten weiter zunehmen wird. Die Hochrechnungen der Patientenzahlen bis zum Jahr 2040 zeigen einen Anstieg der dialysepflichtigen Patienten um rund ein Fünftel. Insbesondere für dialysepflichtige Patienten in Pflegeheimen prognostiziert die Berechnung einen beträchtlichen Anstieg um 37-44\%. 
Die durchschnittlichen Versorgungskosten für chronisch dialysepflichtige Patienten betrugen im Jahr 2017 rund $54200 €$. Für die GKV ergeben sich damit Gesamtkosten von ca. 4,73 Milliarden $€$. Die durchschnittlichen Versorgungskosten für chronisch dialysepflichtige Patienten im Heim waren mit $57200 €$ rund $6 \%$ höher im Vergleich zu Patienten, die nicht im Heim lebten; deren Versorgungskosten beliefen sich auf rund $54000 €$. Dieser Kostenunterschied ist wahrscheinlich darauf zurückzuführen, dass die dialysepflichtigen Patienten im Heim im Mittel älter waren und eine höhere Morbidität aufwiesen, was sich in den Versorgungsaufwänden und -kosten widerspiegelt.

Ein Vergleich der Kosten mit anderen Untersuchungen ist aufgrund abweichender Studiendesigns nur eingeschränkt möglich. Eine Studie aus Deutschland weist für das Jahr 2006 durchschnittliche Dialysekosten von $54777 €$ je Patient aus [5], was unter Berücksichtigung der tatsächlichen jährlichen Inflation in Deutschland Kosten in 2017 von $63601 €$ entsprechen würde. In dieser Studie wurden die Kosten von N = 344 dialysepflichtigen Patienten aus einem Dialysezentrum aus Perspektive der GKV analysiert. Die Altersspanne betrug 22-93 Jahre, der Anteil der männlichen Patienten lag bei $54 \%$ und war damit etwas niedriger als in der hier durchgeführten Analyse. Eine weitere Studie aus Deutschland errechnete durchschnittliche Kosten für das Jahr 2002 von $53613 €$ je Patient [6]. Dies entspricht unter Berücksichtigung der Inflation durchschnittlichen Kosten von $65881 €$ im Jahr 2017. Für die Kalkulation wurden verschiedene Quellen mit Sekundärdaten genutzt (u. a. Statistisches Bundesamt, OECD, publizierte Studien). Die durchschnittlichen Kosten aus beiden Analysen weichen von den in dieser Studie berechneten Werten ab. Dies kann auf die unterschiedliche Studienmethodik zurückgeführt werden, darauf, dass sich Abrechnungsmodalitäten der Leistungen für Dialysebehandlungen und Rahmenbedingungen zur Finanzierung im Gesundheitssystem (z. B. Beträge für EBM-Ziffern) in den letzten Jahren wesentlich geändert haben oder auf andere Behandlungsmodalitäten durch z. B. aktualisierte Leitlinien. Studien aus Deutschland zu den Kosten von chronisch dialysepflichtigen Patienten in Pflegeheimen konnten nicht identifiziert werden, sodass hierzu keine Vergleichswerte vorliegen.

Die beiden wesentlichen Formen der Dialyse bei chronisch Dialysepflichtigen sind die HD und die PD. Bei Patienten aus dieser Analyse wurde am häufigsten die HD eingesetzt, die bei über $90 \%$ der Patienten sowohl innerhalb als auch außerhalb einer stationären Pflegeeinrichtung durchgeführt wurde. Diese Ergebnisse stimmen im Wesentlichen mit internationalen Studien überein, wonach die HD nach wie vor bei der Mehrzahl der Patienten eingesetzt wird. In Europa (Daten ohne Deutschland) erhielten 2014 rund 8 \% der chronisch Dialysepflichtigen eine PD und $92 \%$ eine HD, wenn die Patienten mit Transplantat herausgerechnet werden [28]. Der geringe Einsatz der PD, insbesondere bei Patienten in stationären Pflegeeinrichtungen, ist überraschend, weil diese Form der Dialyse in der Pflegeeinrichtung erbracht werden könnte, ohne den regelmäßigen zeitaufwändigen Transport in ein Dialysezentrum. Aufgrund der häufig körperlichen und kognitiven Einschränkungen der Heimbewohner ist insbesondere die assistierte PD im Setting Pflegeheim umsetzbar [29]. In einer Befragung deutscher Nephrologen bezeichneten fast alle Befragten die Heimdialyse außerhalb eines Zen- trums als sinnvolle Option und rund $93 \%$ präferierten die PD über die HD [30]. Aus den durchgeführten Analysen wird nicht ersichtlich, welche die Gründe für dieses Ungleichgewicht sind. Nephrologen nennen als Barrieren für eine PD außerhalb eines Zentrums insbesondere eine unzureichende Vergütung und die Qualifikation des Personals zur Durchführung der PD [30]. Studien zeigen, dass die PD gegenüber der HD keine Nachteile hinsichtlich der Mortalität [31, 32] oder der Lebensqualität [33] aufweist, stattdessen jedoch weitaus kostengünstiger ist [34, 35]. Die Ergebnisse der vorgenommenen Analysen zeigten ebenfalls, dass die PD kostengünstiger als die HD ist. Vor dem Hintergrund ansteigenden Fallzahlen chronisch Dialysepflichtiger in den kommenden Jahren, v. a. in stationären Pflegeeinrichtungen, bietet die PD eventuell eine Möglichkeit zur Optimierung der Dialyseversorgung und Kostensenkung.

\section{Limitationen}

Folgende Limitationen müssen bei der Interpretation der Studienergebnisse berücksichtigt werden: Die analysierten Daten wurden zu Abrechnungszwecken erhoben. Mögliche Dokumentationsfehler bei der Abrechnung könnten die Ergebnisse verzerren. Allerdings zeigt ein Vergleich mit den Daten der QSD-RL eine nur sehr geringe Abweichung [14], sodass von der Reliabilität der Abrechnungsdaten ausgegangen werden kann.

Für die Hochrechnung der Prävalenz bis 2040 wurden 2 Szenarien mit unterschiedlichen Annahmen berechnet, jedoch können nicht alle relevanten Variablen identifiziert und hinreichend quantifiziert (z. B. medizinischer Fortschritt) werden, die die zukünftige Entwicklung beeinflussen. Es besteht daher die Möglichkeit einer abweichenden Entwicklung der Prävalenz.

Es konnten nur solche Kosten berücksichtigt werden, die über die GKV abgerechnet werden. Die Inanspruchnahme alternativer Behandlungen, welche nicht im Leistungsspektrum der GKV enthalten sind, wurden nicht erfasst. Dies betrifft OTC-Medikamente, private Zuzahlungen der Studienteilnehmer oder Fälle, in denen andere Sozialversicherungsträger Kosten übernahmen.

Es wurden Patienten in die Analysen eingeschlossen, deren Dialysebehandlung über eine EBM-Ziffer abgerechnet wurde. Stationär oder teilstationär erbrachte Dialysen im Rahmen von Krankenhausbehandlungen wurden nicht berücksichtigt. Daher ist es möglich, dass die Prävalenz der chronischen Dialysepflicht unterschätzt wurde. Allerdings ist wichtig anzumerken, dass die Dialyse von Patienten mit chronischem Nierenversagen fast ausschließlich ambulant erfolgt; Dialysebehandlungen im stationären Setting erfolgen meist aufgrund eines akuten Nierenversagens.

Die Hochrechnung der Prävalenz des dialysepflichtigen chronischen Nierenversagens auf die Wohnbevölkerung der BRD erfolgte auf Grundlage der altersspezifischen Prävalenzraten der GKVPopulation, da entsprechende Prävalenzraten für die PKV-Population nicht verfügbar sind. Falls wesentliche Unterschiede im Auftreten des chronischen Nierenversagens zwischen GKV- und PKV-Versicherten bestehen, könnte dies in einer Über- oder Unterschätzung der Prävalenz des dialysepflichtigen chronischen Nierenversagens für die Wohnbevölkerung Deutschlands (GKV und $\mathrm{PKV}$ ) resultieren. 


\section{Schlussfolgerungen}

Zur Prävalenz des chronischen dialysepflichtigen Nierenversagens für die BRD standen bislang keine Daten zur Verfügung, weil Deutschland über kein nationales Register für Nierenerkrankungen verfügt. In dieser Studie wurde die Prävalenz für Deutschland auf einer aktuellen Datengrundlage berechnet. Mit 1210 pmp bewegen sich die Ergebnisse im Rahmen von Daten anderer europäischer Länder. Es wird ein beträchtlicher Anstieg der Dialysepflichtigen bis 2040 prognostiziert, insbesondere für jene die im Heim leben. Hierdurch ist mit weiteren Kostensteigerungen zu rechnen. Die durchschnittlichen Versorgungskosten für dialysepflichtige Patienten betragen jährlich rund $54000 €$. Die häufigste Dialyseform ist die $\mathrm{HD}$, sowohl bei Patienten innerhalb als auch außerhalb stationärer Pflegeeinrichtungen. Die PD wird in nur sehr geringem Maße eingesetzt. Aufgrund der zu erwartenden steigenden Fallzahlen v. a. von Patienten im Heim, könnte die assistierte PD in stationären Pflegeeinrichtungen eine Alternative zur HD aufgrund des Vorteils der ortsunabhängigen Dialyse sein.

\section{Interessenkonflikt}

Die Durchführung dieser Studie wurde von der Baxter Deutschland $\mathrm{GmbH}$ finanziell unterstützt. Die Autoren hatten jederzeit die vollständige Kontrolle über die Durchführung der Studie (Vorgehensweise bei der Analyse, Interpretation der Daten, Verfassen des Manuskripts).

\section{References}

[1] GBD 2017 Disease and Injury Incidence and Prevalence Collaborators Global, regional, and national incidence, prevalence, and years lived with disability for 354 diseases and injuries for 195 countries and territories, 1990-2017: a systematic analysis for the Global Burden of Disease Study 2017. Lancet 2018; 392: 1789-1858

[2] Foreman KJ, Marquez N, Dolgert A et al. Forecasting life expectancy, years of life lost, and all-cause and cause-specific mortality for 250 causes of death: reference and alternative scenarios for 2016-40 for 195 countries and territories. Lancet 2018; 392: 2052-2090

[3] Kidney Disease: Improving Global Outcomes (KDIGO) CKD Work Group KDIGO 2012 Clinical Practice Guideline for the Evaluation and Management of Chronic Kidney Disease. Kidney International Supplements 2013; 3: 1-150

[4] Vanholder R, Davenport A, Hannedouche T et al. Reimbursement of Dialysis: A Comparison of Seven Countries. J Am Soc Nephrol 2012; 23: 1291-1298

[5] Icks A, Burkhard H, Gandjour A et al. Costs of dialysis - a regional population-based analysis. Nephrol Dial Transplant 2010; 25 : 1647-1652

[6] Kleophas W, Reichel H. International study of health care organization and financing: development of renal replacement therapy in Germany. Int J Health Care Finance Econ 2007; 7: 185-200

[7] Kraus D, Wanner C. Epidemiologie chronischer Nierenerkrankungen - werden es immer mehr Patienten? Dtsch Med Wochenschr 2017; 142: $1276-1281$

[8] Denkinger MD, Franke S, Rapp K et al. Accelerometer-based physical activity in a large observational cohort - study protocol and design of the activity and function of the elderly in Ulm (ActiFE UIm) study. BMC Geriatr 2010; 10: 50
[9] Löw M, Stegmaier C, Ziegler H et al. Epidemiologische Studie zu Chancen der Verhütung, Früherkennung und optimierten Therapie chronischer Erkrankungen in der älteren Bevölkerung. Dtsch med Wochenschr 2004; 129: 2643-2647

[10] Holle R, Happich M, Löwel H et al. KORA - a Research Platform for Population Based Health Research. Gesundheitswesen 2005; 67: S19-S25

[11] Völzke H, Alte D, Schmidt CO et al. Cohort Profile: The Study of Health in Pomerania. Int J Epidemiol 2011; 40: 294-307

[12] Brück K, Stel VS, Gambaro G et al. CKD Prevalence Varies across the European General Population. J Am Soc Nephrol 2016; 27: 2135-2147

[13] Aumann N, Baumeister SE, Rettig R et al. Regional Variation of Chronic Kidney Disease in Germany: Results From Two Population-Based Surveys. Kidney Blood Press Res 2015; 40: 231-243

[14] Potthoff F, Weber W, Berendes A et al. Zusammenfassender Jahresbericht der Berichtersteller 2017 - Version 2.0. MNC - Medical Netcare $\mathrm{GmbH} ; 30.06 .2018$

[15] Prakash S, O'Hare AM. Interaction of Aging and CKD. Semin Nephrol 2009; 29: 497-503

[16] Hall RK, Landemann LR, O’Hare AM et al. Chronic Kidney Disease and Recurrent Falls in Nursing Home Residents: A Retrospective Cohort Study. Geriatr Nurs 2015; 36: 136-141

[17] Hoffmann F, Boeschen D, Dörks M et al. Renal Insufficiency and Medication in Nursing Home Residents. Dtsch Arztebl Int 2016; 113: 92-98

[18] Robinson B, Artz AS, Culleton B et al. Prevalence of anemia in the nursing home: contribution of chronic kidney disease. J Am Geriatr Soc 2007; 55: 1566-1570

[19] McClellan WM, Resnick B, Lei L et al. Prevalence and severity of chronic kidney disease and anemia in the nursing home population. J Am Med Dir Assoc 2010; 11: 33-41

[20] Gordon AL, Franklin M, Bradshaw L et al. Health status of UK care home residents: a cohort study. Age and Ageing 2014; 43: 97-103

[21] Hartmann B, Czock D, Keller F. Arzneimitteltherapie bei Patienten mit chronischem Nierenversagen. Dtsch Arztebl Int 2010; 107: 647-656

[22] Scholz S, Biermann-Stallwitz ], Brettschneider C et al. Standardisierte Kostenberechnungen im deutschen Gesundheitswesen: Bericht der Arbeitsgruppe „Standardkosten“ des Ausschusses „ökonomische Evaluation “ der dggö. Gesundheitsökonomie \& Qualitätsmanagement 2020; 25: 52-59

[23] Wissenschaftliches Institut für Gesundheitsökonomie und Gesundheitssystemforschung (WIG2). Analysetools, WIG2 Forschungsdatenbank https://www.wig2.de/analysetools/wig2-forschungsdatenbank. html [abgerufen am: 15.05.2020]

[24] Elixhauser A, Steiner C, Harris R et al. Comorbidity measures for use with administrative data. Med Care 1998; 36: 8-27

[25] Bundesministerium für Gesundheit. Mitglieder und Versicherte der Gesetzlichen Krankenversicherung (GKV), KM 6-Statistik https://www. bundesgesundheitsministerium.de/themen/krankenversicherung/ zahlen-und-fakten-zur-krankenversicherung.html [abgerufen am: 09.09.2019]

[26] Statistisches Bundesamt Bevölkerung Deutschlands bis 2060. Ergebnisse der 13. Koordinierten Bevölkerungsvorausberechnung. Statistisches Bundesamt. Wiesbaden: 2015

[27] Pippias M, Jager KJ, Kramer A. The changing trends and outcomes in renal replacement therapy: data from the ERA-EDTA Registry. Nephrol Dial Transplant 2016; 31: 831-841

[28] Pippias M, Kramer A, Noordzij M. The European Renal Association European Dialysis and Transplant Association Registry Annual Report 2014: a summary. Clinical Kidney Journal 2017; 10: 1-16

[29] lyasere O, Brown E. Dialysis in the nursing home: Caring for patients with ESRD. Journal of Renal Nursing 2014; 6: 126-129 
[30] Pommer W, Wagner S, Müller D et al. Attitudes of nephrologists towards assisted home dialysis in Germany. Clinical Kidney Journal 2018; 11: 400-405

[31] Lukowsky LR, Mehrotra R, Kheifets L et al. Comparing Mortality of Peritoneal and Hemodialysis Patients in the First 2 Years of Dialysis Therapy: A Marginal Structural Model Analysis. Clin J Am Soc Nephrol 2013; 8: 619-628

[32] Heaf JG, Lokkegard H, Madsen M. Initial survival advantage of peritoneal dialysis relative to haemodialysis. Nephrol Dial Transplant 2002; 17: 112-117
[33] Zazzeroni L, Pasquinelli G, Nanni E et al. Comparison of Quality of Life in Patients Undergoing Hemodialysis and Peritoneal Dialysis: a Systematic Review and Meta-Analysis. Kidney Blood Press Res 2017; 42: 717-727

[34] Treharne C, Liu X, Arici M et al. Peritoneal Dialysis and In-Centre Haemodialysis: A Cost-Utility Analysis from a UK Payer Perspective. Appl Health Econ Health Policy 2014; 12: 409-420

[35] Berger A, Edelsberg J, Inglese GW. Cost Comparison of Peritoneal Dialysis Versus Hemodialysis in End-Stage Renal Disease. Am J Manag Care 2009; 15: 509-518 